\title{
¿La psicoterapia transforma la praxis moral?
}

\author{
Mikel Garcia Garcia ${ }^{1,2,3}$ \\ ${ }^{1}$ Médico. Psicólogo. Psicoanalista junguiano. \\ ${ }^{2}$ Cursando doctorado en Estudios Internacionales de Paz, Conflictos y Desarrollo en la \\ Universitat Jaume I (UJI Castellón) \\ ${ }^{3}$ Miembro de la Sociedad Internacional para el desarrollo del Psicoanálisis Junguiano. \\ http://sidpaj.es/
}

Correspondencia mikel@ibiltarinekya.com

Copyright $\odot$ Mikel Garcia 2017. Artículo de acceso abierto bajo la Licencia de Creative Commons. Permite el uso, distribución y reproducción sin restricciones, siempre y cuando la obra original esté debidamente citada.

\begin{abstract}
Resumen
Esta investigación la realicé para responder la pregunta del título partiendo de la hipótesis nula $H_{0}$ : Realizar psicoterapia no se asocia a elecciones utilitaristas ante dilemas morales. Los sujetos participaron voluntariamente, muchos por internet, evaluando unos dilemas éticos, aportando datos sociodemográficos y de religión, y contestando el cuestionario de personalidad MCMI. El análisis es cualitativo (Atlas.ti) y cuantitativo (SPSS) sobre una muestra válida de 85 sujetos. Resultados: a) se rechaza la $H_{0}$. Hacer psicoterapia si está asociada a elecciones utilitaristas; b) los sujetos de más edad son más utilitaristas; c) las personalidades compulsivas con sentimiento de culpa hacen elecciones deontologistas. Se discute sobre los resultados, insuficiencias y alcance de la investigación. Se concluye con una discusión teórica general sobre el interés de seguir investigando, con una perspectiva consiliente y transformadora de la violencia, anticipando áreas a tener en cuenta.
\end{abstract}

Palabras clave: dilemas morales; psicoterapia psicoanalítica junguiana; culpabilidad; desarrollo moral; consiliencia

\begin{abstract}
This research was performed to answer the question of the title from the null hypothesis HO: Performing psychotherapy is not associated with utilitarian choices in the face of moral dilemmas. Subjects participated voluntarily, many online, evaluating ethical dilemmas, providing sociodemographic and religious data, and responding to the MCMI personality questionnaire. The analysis is qualitative (Atlas.ti) and quantitative (SPSS) on a valid sample of 85 subjects. Results: a) $\mathrm{HO}$ is rejected. Do psychotherapy if it is associated with utilitarian choices; $B$ ) older subjects are more utilitarian; C) Compulsive personalities with guilty feelings make deontological elections. The results, shortcomings and scope of the research are discussed. It concludes with a general theoretical discussion about the interest of continuing to investigate, with a consilient and transforming perspective of violence, anticipating areas to take into account.
\end{abstract}

Keywords: moral dilemmas; Jungian psychoanalytic psychotherapy; culpability; Moral development; Consilience

\section{Introducción}

En las terapias psicoanalíticas se constata que el analizando transforma su estructura cognitiva junto a la extinción de los síntomas clínicos. Todos los síntomas, pero alguno más claramente (cleptomanía), conllevan conductas amorales. La extinción de la patología dota al sujeto, al liberarlo del determinismo, de un potencial de funcionamiento moral distinto. No es habitual, en la clínica, evaluar la cognición moral en las altas terapéuticas. Pero en mi experiencia hay datos que ponen en duda que las decisiones morales sean distintas. Clarificar esto requiere investigación en un marco distinto del clínico. 
En la revisión bibliográfica constato que la filosofía se ha ocupado más que la psicología. Hay dos posiciones contrastadas en el entendimiento clásico de la moralidad. La deontológica postula que ciertas acciones son siempre amorales, independientemente de cuán buenas sean las intenciones o resultados y que las decisiones morales se basan en respuestas innatas, automáticas, intuitivas y más emocionales (Kant, 2008; Scanlon, 1982, 1998). La utilitaria o consecuencialista (Mill, 1989; Regan, 1980; Elster \& Grapes, 1982; Bentham, 1983) postula que la acción correcta es la que provoca un bien mayor, independientemente de los medios utilizados y que las elecciones se basan en un razonamiento de deliberación evaluativa más lenta que conlleva esfuerzo. Una situación típica de elección utilitarista es sacrificar un sujeto para salvar un grupo. Alasdair Maclntyrel, principal exponente de la "nostalgia comunitarista", en After virtue (MacIntyre, 1981) hace un lúcido estudio histórico de la ética que concluye en un diagnóstico pesimista: la ética ya no es posible puesto que no es posible llegar a acuerdos morales ni fundamentarlos racionalmente. Cada sujeto se adscribe a una u otra forma de ética según la coherencia entre sus "atractores" internos y las ofertas externas: sus elecciones son relativistas. Cercana a esa posición relativista, más intersubjetiva, está la teoría diádica (Gray, Waytz, \& Young, 2012) que proporciona un marco en el que la cognición moral probablemente no se caracteriza por el conflicto entre las acciones de "agentes" y los resultados en "pacientes", sino por su activación mutua. Teoría también cuestionada por investigaciones (Baxter, 2016) que apuntan a una prevalencia de la "percepción de la mente". Para Kant los seres humanos nos caracterizamos por una "insociable sociabilidad" (Martínez Guzmán, 1997). Haidt y Joseph (2007) intentaron identificar las bases psicológicas mediante las cuales las diferentes culturas crean la gran variedad de sistemas morales. Encontraron cinco: Daño, Justicia y equidad, Filiación y lealtad, Autoridad y respeto, Pureza y santidad. Hay investigaciones controvertidas de la neurociencia. Greene et al., (2001; 2004) encuentras diferencias en las evaluaciones morales de pacientes con daño en la Corteza Prefrontal Ventromedial -CPV y personas sin ningún daño neurológico. La hipótesis del "paciente utilitarista" (de moda en la neurociencia cognitiva), que tienen lesión en CPV y muestran una disposición utilitarista en sus respuestas a los "dilemas morales personales" revelando una posible carencia de empatía. Hay pocos estudios basados en rasgos de personalidad. Una realizada con psicópatas reveló que un subgrupo de estos (con poca ansiedad) hacía elecciones claramente utilitaristas (Koenigs, Kruepke, Zeier, \& Newman, 2012).

De esta revisión se infiere que hay más literatura sobre la discusión entre deontologistas y utilitaristas, que sobre la intersubjetividad; y que la utilitarista se asocia a lesiones, a psicopatías... Alguno afirma que el hecho de que la mayor parte de las personas suelan preferir la opción deontologista indica que ha sido favorecida por la selección natural que busca maximizar la cooperación.

En esta investigación exploro el funcionamiento moral con una perspectiva enriquecida con datos acerca de la estructura de la personalidad y con una metodología de análisis cualitativo cuantitativo. Mayntz, Holm \& Hübner (1969) plantean que la diferencia entre lo cuantitativo y lo cualitativo es poco precisa. Resulta imposible que un investigador pueda recurrir a una forma sin utilizar la otra. El Enfoque Integrado Multimodal, también insiste en la integración de lo cuantitativo y cualitativo Henwood (2004, 2008). Formular una hipótesis promueve rigurosidad en la investigación, voy a establecer como hipótesis nula $\mathrm{H}_{0}$ : Realizar psicoterapia no se asocia a elecciones utilitaristas ante dilemas morales. 


\section{Método}

Participantes, procedimiento

Procedencia de la muestra. Dos vías: a) contacto directo con participantes de actividades sociales propias como seminarios, conferencias; b) mediante internet. Publiqué en mis redes el proyecto de investigación y fue contestado por bastantes sujetos. Las muestras de internet son frecuentemente usadas en la investigación psicológica ya que mantienen la confiabilidad igual a las poblaciones basadas en laboratorio mientras que proporcionan una mayor diversidad (Skitka \& Sargis, 2006). Los sujetos participaron voluntariamente y sin remuneración (el sistema MTurk si remunera). Total 499 protocolos.

Material

Los participantes rellenaron un material. La mayoría electrónicamente mediante formularios "docs" depositados en mi espacio google. Secciones del material: a) sociodemográfica; b) religión; c) cuestionario de personalidad MCMI (Inventario Clínico Multiaxial de MILLON). El anexo 1 presenta un informe ficticio del MCMI; d) ocho dilemas morales con dos respuestas una obligatoria (si/no) y otra voluntaria, consistente en relatar las razones, los sentimientos, reflexiones, que le producía el dilema... El anexo 2 contiene los dilemas usados en la investigación en el orden en que se presentaban. He excluido los que diseñé para explorar actitudes como la confianza, la "lealtad invisible" y la paranoia.

En su diseño controlé varias variables: a) orden de presentación; b) tipo de material; c) que recogieran aspectos que se discuten en las investigaciones sobre la moralidad; d) que abordasen situaciones factibles de estar cercanas a la experiencia de la gente en la actualidad y e) que promovieran contacto emocional y reflexión introspectiva en los participantes. Los dilemas tienen una naturaleza paradójica que los hace evocadores emocionalmente (Greene et al., 2001).

¿Qué áreas exploran?

El dilema del moribundo (3) la dicotomía "personal" Deontologismo/Utilitarismo. Este es el principal en la investigación. Lo presento en tercer lugar y mediante una imagen, para que emerja en un contexto de remoción emocional por los anteriores y convoque una respuesta inmediata sin el freno de tener que comprender un texto.

El dilema "gratificación" (1) obliga a elegir una modalidad "placentera" en un contexto subyacente de dimensiones polares: intima/social y secreto/divulgación.

El dilema "demanda de dolor sexual" (2), confronta al sujeto con actuar un daño consentido. Un modo de dicotomía Deontologismo/Utilitarismo indirecta o "impersonal".

El dilema "perdón" (4) confronta con actuar desde el resentimiento o desde la compasión. En "Libertad y resentimiento" Strawson (1995) diserta acerca de ello.

El dilema "autorizar inseminación" (ㄱ) confronta con la responsabilidad de sancionar el deseo de otro que demanda con cierta propiedad respecto a un tercero muerto.

El dilema "ecológico" (8) confronta el altruismo con el propio interés.

El conjunto contiene los factores recogidos por Haidt y Joseph.

Criterios de exclusión. He excluido los protocolos que no estaban totalmente cumplimentados y los que no cumplían los criterios de validez y fiabilidad del MCMI, resultando una muestra válida de 85 sujetos (17\%).

\section{Análisis}

Categorizaciones de la muestra

1.- Basándome en el "dilema del moribundo" y contrastando la descripción abierta con su respuesta si/no, he codificado los sujetos como pertenecientes a una de estas tres 
categorías: deontologistas; utilitaristas y relativistas. La relativista es equivalente a la "Utilitarista_Deontologista" de la investigación de Everett et al (2016).

2.- Con las respuestas de la sección de religión, he codificado los sujetos como pertenecientes a una de estas categorías: ninguna religión; espiritualidad; ateo/agnóstico; budista; monoteísta/católica.

3.- Según el resultado de la corrección del MCMI he codificado los sujetos como pertenecientes a una de estas categorías: sano; neurótico; fronterizo; psicótico.

La categorización genera tres nuevas variables discriminativas a incluir para el análisis cuantitativo que he realizado mediante el programa estadístico SPSS.

He utilizado la herramienta Atlas.ti para el análisis cualitativo con el fin de descubrir las redes semánticas subyacentes en las respuestas explicativas voluntarias de todos los dilemas morales propuestos en la investigación.

\section{Resultados}

Del total de la muestra

La mayor parte de las respuestas al dilema del moribundo son deontologistas, le siguen las utilitaristas y por último las relativistas. Las mujeres son más relativistas.

\begin{tabular}{lc|c} 
& Frecuencia & Porcentaje \\
\hline Utilitaristas & 24 & 28,2 \\
\hline Relativistas & 9 & 10,6 \\
\hline Deontologistas & 52 & 61,2 \\
\hline Total & 85 & 100,0 \\
\hline
\end{tabular}

Tabla 1 Tipos elecciones morales

\begin{tabular}{lc|c|c} 
& Utilitaristas & Relativistas & Deontologistas \\
\hline Hombre & $41,7 \%$ & $33,3 \%$ & $42,3 \%$ \\
\hline Mujer & $58,3 \%$ & $66,7 \%$ & $57,7 \%$ \\
\hline
\end{tabular}

Tabla 2 Tipos elecciones morales según género

En la llustración 1, se presenta la red "tirar al moribundo" obtenida tras el análisis con Atlas.ti.

No tirar al moribundo es la elección de los deontologistas que está: 1. determinada por la espiritualidad asociada a no tener la potestad de controlar el destino; 2 . causada por no poder soportar en el futuro el sentimiento de culpa y por la identificación con el moribundo especialmente si es conocido o familiar; 3. favorecida por la presión del grupo; 4. asociada a una actitud de sacrificio por ejemplo tirándose el sujeto al agua para dejar sitio al moribundo; 5. asociada a acompañar al muriente en su proceso de muerte cuidándolo; 6. asociada a la esperanza en que ocurra algo que salve la situación, 7 . asociada a la necesidad de que la decisión sea consensuada por el grupo; 8. asociada con el canibalismo, consistente en no tirar al moribundo y tenerlo como reserva para poder comerlo si el naufragio dura y no son rescatados.

Tirar al moribundo para los utilitaristas: 1. no está impedida por ser conscientes de que ese acto sería un hecho que les causara culpa en el futuro; 2 . asociado con sentimiento de dolor por la decisión; 3. asociada con indagar si hay otros candidatos; 4. asociada con la identificación y actitud de acompañamiento al moribundo; 5. el sacrificio es parte de esa actitud, uno siente que sacrifica algo. Tirar al moribundo para los relativistas está asociado con: 1 . hacerlo a un modo de eutanasia; 2 . tener una actitud ecológica; 3. con una actitud caníbal. 


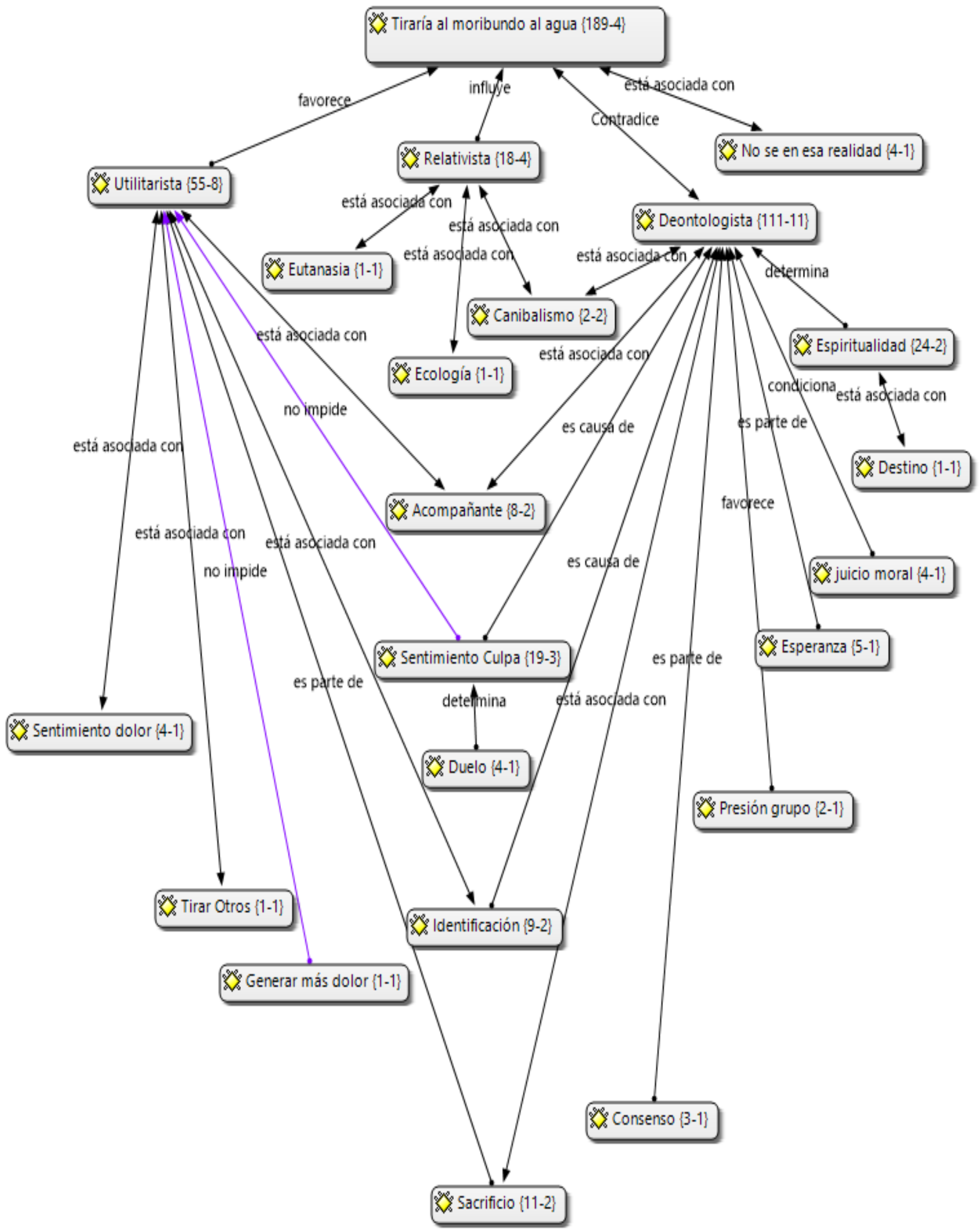

Ilustración 1 Red "Tirar al moribundo" Atlas.ti Clasificación topológica. Se indican las relaciones y los tipos de asociación de los conceptos codificados: asociación; determinación; favorece; influye... Los números entre paréntesis indican etiquetas en las categorizaciones del Atlas.ti 
Los deontologistas no tirarían al moribundo al agua, no accederían a causar dolor a su pareja, ni a perdonar su infidelidad, denunciarían a la empresa y preferirían tener sexo. Los utilitaristas tirarían al moribundo, denunciarían a la empresa, y no accederían a causar dolor a su pareja. Los relativistas tirarían al moribundo, preferirían tener sexo, y denunciarían a la empresa. Todos los grupos autorizarían inseminación artificial (tabla 3).

\begin{tabular}{|c|c|c|c|c|}
\hline & & $\begin{array}{l}\text { Utilitaristas } \\
\% \text { columnas }\end{array}$ & $\begin{array}{l}\text { Relativistas } \\
\% \text { columnas }\end{array}$ & $\begin{array}{l}\text { Deontologistas } \\
\% \text { columnas }\end{array}$ \\
\hline Gratificación & Tendría sexo & $50,0 \%$ & $55,6 \%$ & $69,2 \%$ \\
\hline Dolor & Causaría dolor & $45,8 \%$ & $22,2 \%$ & $28,8 \%$ \\
\hline Tirar al moribundo & Lo tiraría al agua & $50,0 \%$ & $33,0 \%$ & $11,9 \%$ \\
\hline Resentimiento/compasión & No perdonaría & $58,3 \%$ & $55,6 \%$ & $61,5 \%$ \\
\hline Juez & Autorizaría la inseminación & $79,2 \%$ & $77,8 \%$ & $71,2 \%$ \\
\hline Altruismo/interés propio & Denunciaría a la empresa & $79,2 \%$ & $55,6 \%$ & $71,2 \%$ \\
\hline
\end{tabular}

Tabla 3 Tabla cruzada dilemas*tipos de moralidad

Los que no pertenecen a ningún tipo de religión son más relativistas. Los budistas son los menos utilitaristas. (ilustración 2).

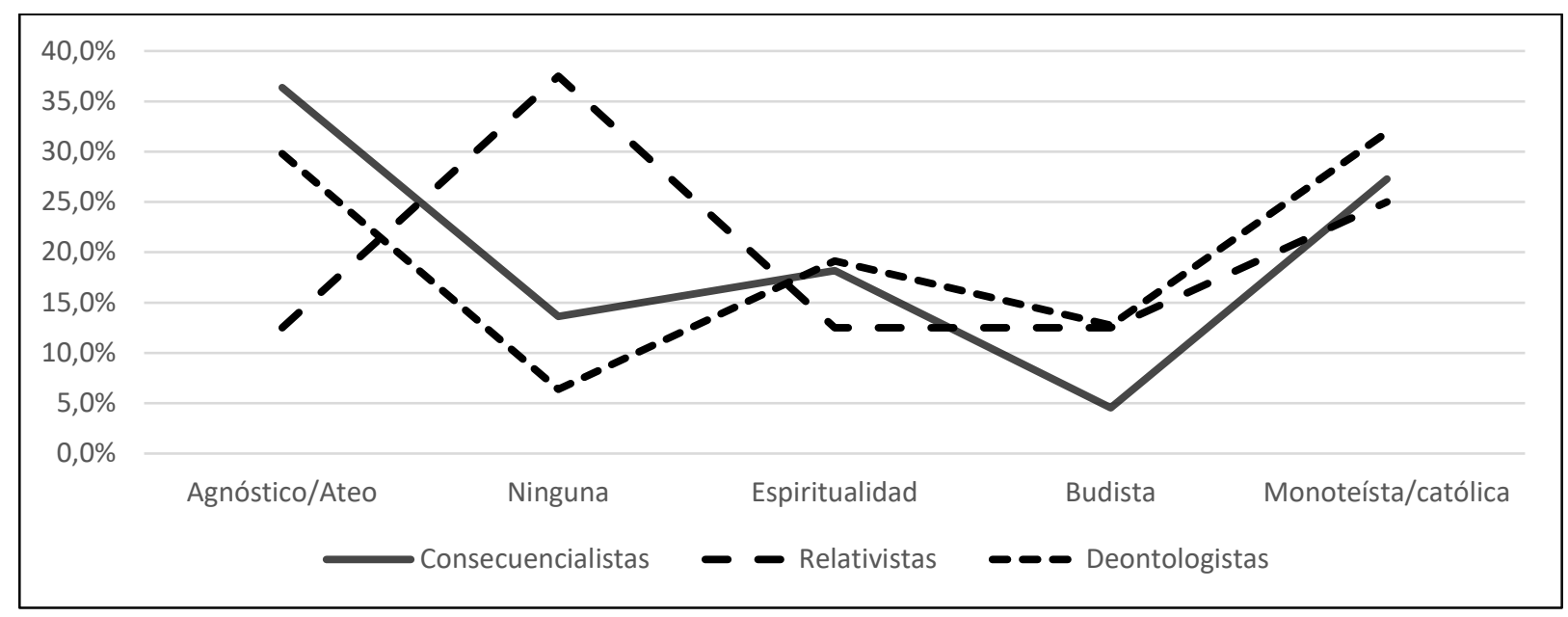

Ilustración 2 Religiosidad y elecciones morales

Para el análisis de los datos de personalidad he buscado verificar la lo encontrado en el análisis del Atlas.ti. La fiabilidad de las escalas del MCMI, en consistencia interna, es buena (alpha de Cronbach es 0,82). Los análisis factoriales MCP han podido hacerse al cumplir los requisitos RMO $(0,829)$ y Bartlett (sig. 0.001). Los resultados se presentan en las tablas 4,5 y 6 e ilustraciones 3 y 4 .

Los datos de relativistas tienen menor consistencia al ser su muestra la más pequeña. Los utilitaristas en relación a los deontologistas tienen un perfil más saludable puntúan más en los patrones de personalidad más sanos y menos en las escalas clínicas, encontrándose un perfil opuestos en distimia, hipomanía, alcoholismo y drogadicción. La puntuación en distimia tiene significatividad estadística en el análisis $t$-test para grupos independientes $(\mu 1 \neq \mu 2)$. [t(59) $=2.45, p=.0312]$. En la ilustración 5 se representa en perfil completo del MCMI de los sujetos deontologistas que explicaron que la culpabilidad que sentirían en el futuro su razón para no tirar al moribundo era. Este perfil se caracteriza por un patrón de personalidad compulsivo-esquizoide-evitante con depresión-ansiosa y contenciones sin compensaciones escapatorias como drogas o alcohol. 


\begin{tabular}{cc|c|c|c}
\multicolumn{2}{c}{ Utilitaristas } & Relativistas & Deontologistas \\
\hline \multirow{3}{*}{$\begin{array}{c}\text { Estructura } \\
\text { Clínica }\end{array}$} & Sano & $16,7 \%$ & $11,1 \%$ & $7,7 \%$ \\
\cline { 2 - 5 } & Neurótico & $58,3 \%$ & $66,7 \%$ & $69,2 \%$ \\
\cline { 2 - 5 } & Fronterizo & $25,0 \%$ & $22,2 \%$ & $19,2 \%$ \\
\cline { 2 - 5 } & Psicótico & $0,0 \%$ & $0,0 \%$ & $3,8 \%$ \\
\hline
\end{tabular}

Tabla 4 Tabla cruzada estructuras de personalidad*tipos de moralidad

\begin{tabular}{|l|r|r|}
\hline Análisis factorial MCMI & Utilitaristas & Deontologistas \\
\hline Factores y varianza explicada & $5 / 86,525 \%$ & $4 / 78,937$ \\
\hline Varianza del primer factor & $31,963 \%$ & $38,958 \%$ \\
\hline Tabla 5 Análisis factorial MCMI
\end{tabular}

\section{Gráfica de variables del tercer factor que discriminan tipos moral}

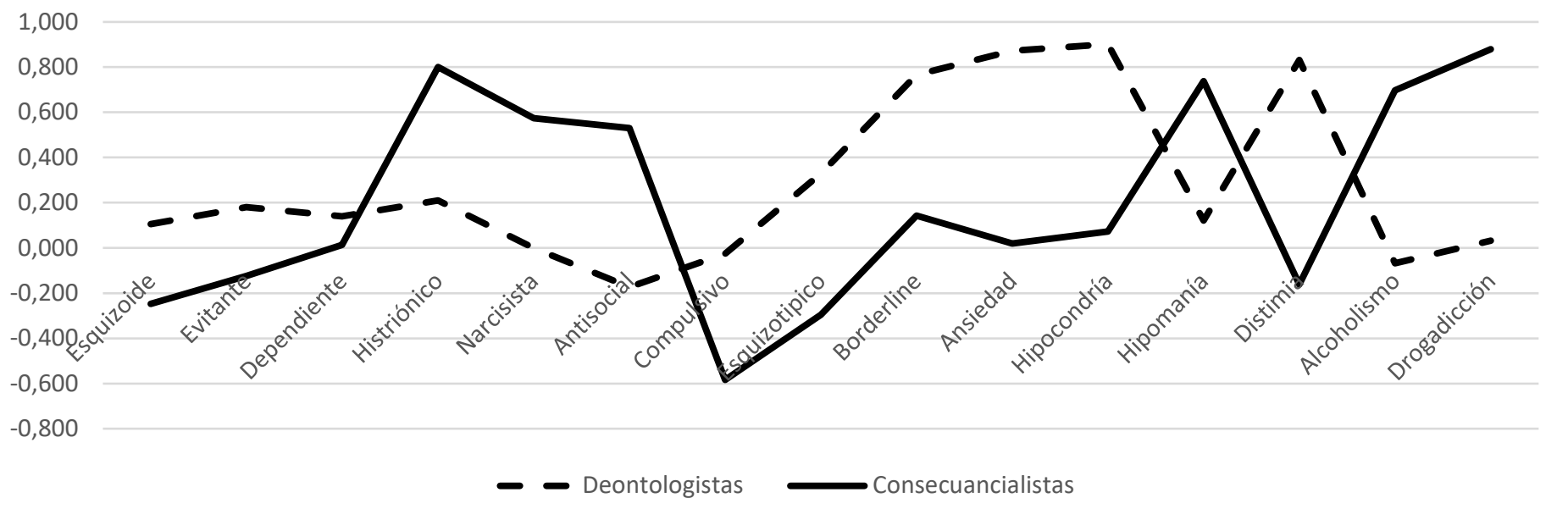

Ilustración 3 Escalas que discriminan los tipos morales en el tercer factor

\begin{tabular}{|l|c|c|}
\hline Escalas MCMI & Utilitaristas & Deontologistas \\
\hline Compulsivo & $-0,314$ & 0,561 \\
\hline Distimia & $-0,162$ & 0,831 \\
\hline
\end{tabular}

Tabla 6 Tabla cruzada peso en factor escalas MCMI*tipos de moralidad

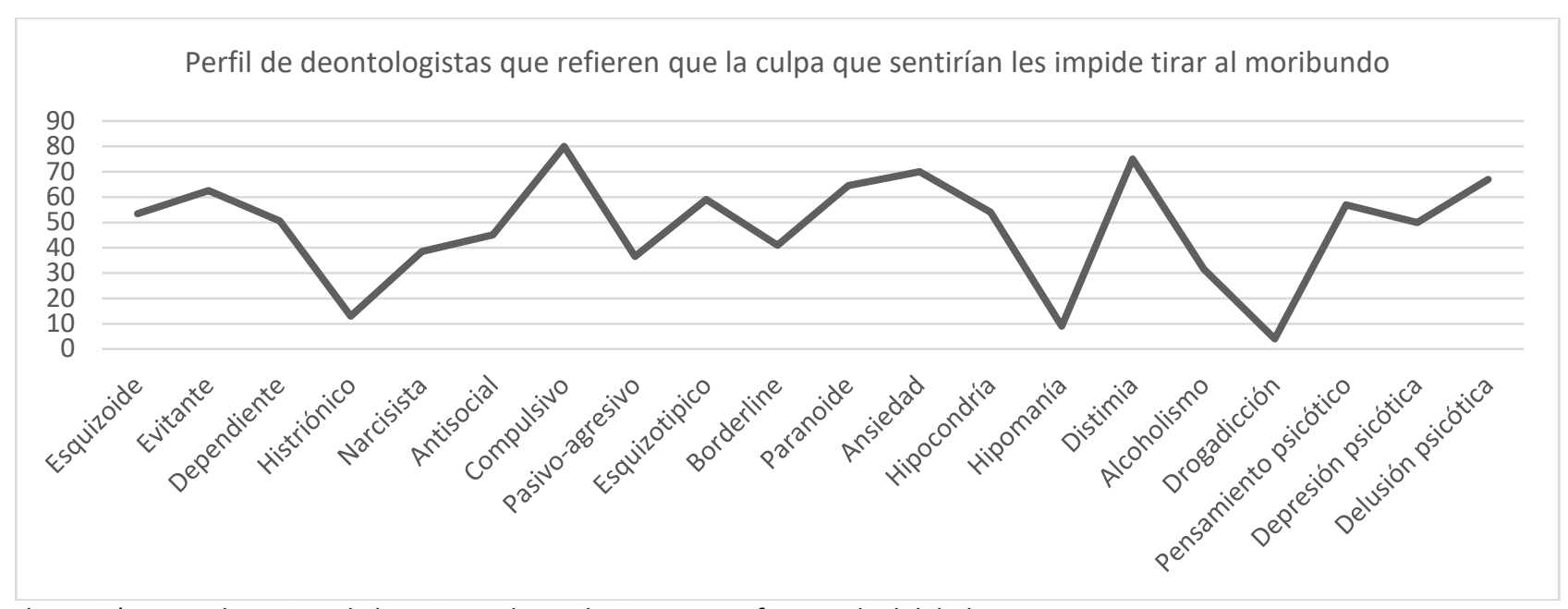

Ilustración 4 Escalas MCMI de los sujetos deontologistas que refieren culpabilidad 
Otra asociación con significatividad estadística: Los utilitaristas tienen más edad. El tes t $(t(74)=3,496, p=.001)$. El F-test ANOVA de una vía $(F(84)=6,341, p=.003)$. Para $M=48,38$ años con un SD (+/-10,842), $M=39,19$ años con un SD $(+/-10,53)$.

Resultados del análisis en relación a la psicoterapia.

Los resultados aparecen recogidos en las tablas 7 a 11.

Los que han hecho psicoterapia, son los más utilitaristas (tabla 7) y este dato tiene significatividad estadística en el análisis Chi Cuadrado. $\left.\left[X^{2}(2, N=85)=9,533, p=.009\right)\right]$. Son el $20 \%$ del total de la muestra, una representación alta comprando con la población general. En las siguientes tablas se aprecian tendencias de este grupo, no estadísticamente significativas: son menos creyentes o de mayor religiosidad y más sanos. Han contestado por igual sobre tirar al agua al moribundo y lo mismo hombres que mujeres. El estudio de sus respuestas descriptivas ha variado su clasificación inicial. El grupo de relativistas ha procedido sobre todo de los inicialmente deontologistas (48\% responden no tirar al agua al moribundo), y esto ha ocurrido más con las mujeres.

\begin{tabular}{||l|r|c|r|c|}
\hline \multirow{2}{*}{ Tipo moral } & \multicolumn{4}{|c|}{ Psicoterapia No /Si } \\
\cline { 2 - 5 } & Casos & $\%$ & Casos & $\%$ \\
\hline Utilitaristas & 16 & $23,5 \%$ & 8 & $47,1 \%$ \\
\hline Relativistas & 5 & $7,0 \%$ & 4 & $28,6 \%$ \\
\hline Deontologistas & 47 & $69,1 \%$ & 5 & $29,4 \%$ \\
\hline Total & 68 & & 17 & \\
\hline
\end{tabular}

Tabla 7 Tabla cruzada Tipos moral*Psicoterapia

\begin{tabular}{|l|r|r|r|}
\hline \multicolumn{1}{|c|}{ Religión } & \multicolumn{2}{|c|}{ Psicoterapia No/Si } & Total \\
\hline Monoteístas & 19 & 1 & 22 \\
\hline Budista & 3 & 1 & 4 \\
\hline Religiosidad & 21 & 9 & 30 \\
\hline No creyente & 21 & 5 & 26 \\
\hline Ateo/agnóstico & 4 & 1 & 5 \\
\hline & 68 & 17 & 85 \\
\hline
\end{tabular}

Tabla 8 Tabla cruzada Religión*Psicoterapia

\begin{tabular}{|l|r|r|r|r|}
\hline \multirow{2}{*}{ Estructura Clínica } & \multicolumn{4}{|c|}{ Psicoterapia No/Si } \\
\cline { 2 - 5 } & Casos & \multicolumn{1}{c|}{$\%$} & Casos & \multicolumn{1}{c|}{$\%$} \\
\hline Sano & 4 & $5,9 \%$ & 5 & $29,4 \%$ \\
\hline Neurótico & 46 & $67,2 \%$ & 10 & $58,6 \%$ \\
\hline Fronterizo & 16 & $23,7 \%$ & 2 & $11,1 \%$ \\
\hline Psicótico & 2 & $2,8 \%$ & 0 & $0,0 \%$ \\
\hline
\end{tabular}

Tabla 9 Tabla cruzada Estructura Clínica*Psicoterapia

\begin{tabular}{|l|r|c|r|c|}
\hline \multirow{2}{*}{ ¿Lo tiraría al agua? } & \multicolumn{4}{|c|}{ Psicoterapia No/ Si } \\
\cline { 2 - 5 } & Casos & \multicolumn{1}{c|}{$\%$} & Casos & \multicolumn{1}{c|}{$\%$} \\
\hline No & 46 & $67 \%$ & 8 & $48 \%$ \\
\hline $\mathrm{Si}$ & 22 & $32 \%$ & 9 & $52 \%$ \\
\hline
\end{tabular}

Tabla 10 Tabla cruzada Dilema moribundo*Psicoterapia 


\begin{tabular}{|l|r|r|r|r|}
\hline \multirow{2}{*}{ ¿Lo tiraría al agua? } & \multicolumn{2}{|c|}{ No Psicoterapia } & \multicolumn{2}{c|}{ Si Psicoterapia } \\
\cline { 2 - 6 } & Hombre & Mujer & Hombre & Mujer \\
\hline No & 19 & 27 & 5 & 5 \\
\hline Si & 9 & 13 & 4 & 3 \\
\hline
\end{tabular}

Tabla 11 Tabla cruzada Dilema moribundo*Psicoterapia*género

El estudio sobre que variables pueden influir en el tipo de elección moral no se ha encontrado que las escalas de personalidad MCMI influyan (análisis de regresión lineal). Sin embargo el análisis de regresión para datos categóricos (escalamiento óptico) testando como predictores psicoterapia, edad y género, si ha encontrado significancia estadística [ANOVA $(F(84)=2,853, p=.011)$ ]. Género no tiene significatividad.

\begin{tabular}{l|c|c|c} 
& $\mathrm{F}$ & Sig. & Importancia \\
\hline Psicoterapia & 7,760 &, 000 &, 629 \\
\hline Edad & 3,192 &, 047 &, 357 \\
\hline Sexo &, 618 &, 542 &, 014 \\
\hline Tabla 12 F, significatividad e importancia
\end{tabular}

\section{Hallazgos y discusión}

Resultados: a) se rechaza la $\mathrm{H}_{0}$. Por lo tanto, hacer psicoterapia si está asociada a elecciones utilitaristas; b) los sujetos de más edad son más utilitaristas; c) las personalidades compulsivas con sentimiento de culpa hacen elecciones deontologistas.

Tiene sentido que la psicoterapia esté asociada a elecciones morales utilitarias ya que el trabajo de introspección genera autoconocimiento, adquisición de un estilo de investigar sobre la conciencia y la capacidad de distanciarse de los automatismos internos que, además, ya no sobredeterminan. Condiciones para poder deliberar sobre el escenario del dilema propuesto. Las descripciones ante los dilemas manifiestan esa deliberación. El proceso de elección es complejo, contempla más dimensiones, es intersubjetivo. Alguno afirmó que en un escenario real quizás hiciese otra cosa. Varios afirmaron que sería doloroso tomar la decisión de tirar al moribundo y que ese dolor duraría. El proceso de psicoterapia acerca a ambos géneros hacia estilos más igualitarios de respuestas, aunque sin significatividad estadística. Que los de más edad destaquen por hacer más elecciones utilitaristas probablemente está influido por la dimensión temporal de la duración de la terapia de los sujetos analizados.

En una investigación con el "dilema del niño llorando" (Greene, 2009) el investigador probó que los impulsos iniciales pueden contenerse con un proceso de deliberación que activa una segunda área cerebral. Dilema: Una madre y su hijo están ocultos junto con otras muchas personas en un sótano, en suelo enemigo en situación de guerra, el llanto del niño alertará a los enemigos que si lo oyen matarán a todos los refugiados. ¿Llegaría a matar al niño si fuese necesario? En todos los sujetos se incrementa la actividad en el córtex cingulado anterior. Cuando la respuesta al dilema es: sí, mataría a mi hijo, se eleva la actividad en el córtex prefrontal dorsolateral, sugiriendo un procesamiento adicional que reemplaza la reacción inicial ante lo impactante del dilema.

El otro hallazgo relevante es la relación entre la elección deontologista y el sentimiento de culpa, que impide tomar la decisión de "dañar" al otro en beneficio del colectivo. Esta asociación tiene significación estadística: un patrón de personalidad compulsivo-esquizoide-evitante con depresión-ansiosa. De lo que se infiere que se trataría de una un sentimiento de culpa obsesivo-rumiadora difícil de confrontar y que podría tener 
matices "persecutorios" generando ansiedad. La elección deontológica en este caso es un mecanismo de evitación que le determina y le impide un ejercicio libre para elegir.

En la discusión moral se discute sobre la existencia de una moral innata cuya función esencial fuera promover el progreso de la especie humana, al contrario de la consideración de la moral como freno al egocentrismo, la falta de empatía, y la cultura de la guerra perpetua. Aunque fuese cierto difícilmente podría actuar en sujetos determinados por el daño (trauma) en su desarrollo. Son sugerentes varias propuestas. Una de ellas, acerca de los componentes instintivos de la reciprocidad (Suchak \& de Waal, 2012) en la que los autores prueban que los monos capuchinos comprenden la ventaja de la reciprocidad y la actúan sin la carga cognitiva negativa (de muchos humanos) que frena la reciprocidad. Cogniciones negativas, y "mecanismos de defensa psicoanalíticos" son denominaciones equivalentes, surgen de un aprendizaje ensayo error como respuesta adaptativa a un medio hostil, y son, también similares a los samskaras indios (Eliade, 1998).

El daño, parece ser un factor determinante, pero no el objetivo sino la percepción subjetiva de quien elige. Incluso el dilema de la gratificación puede ser percibido como daño a un tercero ausente (la pareja actual o edípica) como si fuese una víctima de una injusticia, y esa percepción determina la elección. Por el contrario, se puede dejar de ver el daño en los casos de genocidio (Castano \& Giner-Sorolla, 2006).

No hacer daño es habitualmente un mecanismo de defensa frente a la retaliación (ser dañado), más que una actitud altruista, y si este no es efectivo aparecen otros mecanismos: la negación, la justificación (considerar infrahumanos a los aborígenes australianos citado por Castano), o la escisión. El principio "No hagas a otro lo que no quieras que te hagan a ti" es percibido e interpretado de modo muy diverso según las subjetividades. Algunos sujetos del estudio han recurrido al mismo para no acceder a producir el dolor demandado por su pareja. Es necesaria la empatía para la intersubjetividad, pero algunos sujetos perciben como empatía lo que es un mecanismo defensivo de identificación proyectiva, y eso les lleva a un automatismo deontológico. No hacen daño para no sufrir ellos evitando sentir tanto del dolor del otro. Es el polo opuesto al de los psicópatas que carecen de una respuesta empática al dolor de las víctimas y que podrían hacer elecciones utilitaristas de un modo automático.

La teoría de las fundaciones morales (Graham et al., 2013) asume la ausencia de daño objetivo en las violaciones de la "pureza" (ejemplo: comer un perro muerto). Un acto malo o impuro en la dimensión bien/mal también puede ser percibido como dañar, en última instancia, a uno mismo. Son diversos mecanismos cuya finalidad es evitar ser más dañados. Algunos sujetos han referido la opción de un autosacrificio antes que hacer daño, tirándose al agua en vez de tirar al moribundo. Un fenómeno que puede tener varios significados no explorados en esta investigación; descargar culpa, evitar el castigo (mayor) de otro con un autocastigo "controlado"; complejo de héroe en la polaridad narcisistamasoquista; un acto de pureza. ¿Hacer el bien para evitar el mal mayor? Algunas de estas disposiciones están en el ideal social que premia, y, a veces exige, el autosacrificio en aras del bien.

En el autosacrificio caben seres queridos; la "pureza del sacrificio de Isaac Abraham" es un referente. Dice Derrida que puede hablarse tanto del sacrificio de Abraham como el de Isaac: "... y es éste el sacrificio de los dos, el dar-(la)-muerte-al-otro dándose (la) muerte, mortificándose para dar esta muerte en ofrenda sacrificial a Dios..." (2000, p. 71). Dios da una orden doblemente imposible: por un lado, porque pide lo peor; por otro, porque se retracta. En las autoinmolaciones terroristas pueden converger aspectos de estas dinámicas complejas. Sachdeva (2015) investiga sobre el significado del autosacrificio en el juicio moral. Sobre el suicidio hay discusión entre investigadores 
que afirman que la percepción de daño predice el juicio moral ante el suicidio (Gray, 2014), y otros que responden que la pureza es más importante que el daño (Rottman, Kelemen, \& Young, 2014).

La percepción de daño se sustenta en el sistema castigo/recompensa por los actos morales extendida a otras posibles existencias (karma/reencarnación; cielo/infierno). En última instancia lo que subyace en el núcleo es la tierra tenebrosa de la ansiedad ante la muerte que la Teoría del Manejo del Terror (TMT) considera clave en la dinámica de los sujetos.

Las respuestas deontológicas pueden estar motivadas por la necesidad de conformación social. Un sujeto expresó que eligió pasar un día en vez de acostarse y tener una relación sexual porque pensaba o imaginada que era lo aceptable en la sociedad. Everett (2016) hace una investigación que concluye que las personas que hacen juicios deontológicos son preferidas como agentes sociales, porque son percibidos como más morales y de confianza.

La violencia estructural cultural coloniza la mente con referentes que justifican la violencia. Los intentos individuales de liberación pueden ser fallidos "Kafka: intento fallido de liberarse del colonizador interno" (Bleichmar, 2016). La psicoterapia tiene como objetivo el análisis de los aspectos más íntimos del impacto de la violencia estructural, no aborda la totalidad. El sujeto cambia radicalmente de actitud ante creencias desde un fanatismo hasta una perspectiva de realidad compleja. Aunque capacitado para la praxis de una moralidad más autónoma, sigue manteniendo un alto grado de contingencia a las circunstancias de una situación real. Cuando sea agente activo de la acción moral dependerá de la complejidad del vínculo que establezca con el agente pasivo que la recibe. Esto trasciende la díada a una dimensión transpersonal. La relación cara-a-cara es fundamental para Lévinas. "Soy totalmente solo; así, pues, el ser en mí, el hecho de que existo, mi existir, es lo que constituye el elemento absolutamente intransitivo, algo sin intencionalidad ni relación. Todo se puede intercambiar entre los seres, salvo el existir" (Lévinas, 2000, pp 53 y 54).

\section{Insuficiencias de la investigación}

La inmadurez como investigador, ha propiciado el error de haber dejado como voluntarias las respuestas abiertas reduciendo la muestra válida. Estas respuestas abiertas carecían de estructura bien definida por lo que la clasificación en los tipos morales tuvo su dificultad.

Indeterminación de algunos términos. Psicoterapia no estaba delimitado, no puedo saber ni si la terminaron o estaban haciéndola, ni el tipo de psicoterapia, aunque probablemente es psicoanalítica. En religión no se precisaba si la actual o en la que el sujeto había sido aculturado.

La investigación la he diseñado, ejecutado, analizado y redactado yo mismo.

Varios presupuestos que he utilizado no están verificados. Es discutible que la imagen para el dilema del moribundo, facilite una respuesta más automatizada. Buscaba provocar un heurístico en su acepción de atajo rápido que resolviese el dilema sin deliberar demasiado. Pero depende de la capacidad de simbolización del sujeto.

\section{Discusión general}

Inicialmente el infante humano es "amoral", adquiere una conciencia ética a lo largo de su desarrollo, que puede estar consolidada al final de la adolescencia, como lo indican las investigaciones psicoanalíticas, las clásicas de Piaget o Kholberg, entre otras. Una investigación (Wellman at al., 2001) afirma que las habilidades socio-cognitivas se desarrollan durante los primeros 5 años de vida y se consolidan en la adolescencia. Es 
lo que afirma la investigación psicoanalítica que sitúa la terminación de la fase edípica alrededor de los 5 años. Otra investigación estudia la adolescencia (Blakemore \& Choudhury, 2006) y aporta datos neurocientíficos en la misma línea.

Es interesante comprobar que la investigación sobre la moralidad va propiciando confluencias entre paradigmas y que las restricciones metodológicas del positivismo, el conductismo, y, a veces, el historicismo, que podían paralizar una actitud consiliente, se van relajando, a la par que hay interés en indagar sobre las estructuras ("cajas negras") que dan sentido a los rasgos parciales. Tal y como dice Edgar Morin (1994), el pensamiento complejo está animado por la aspiración a un saber no parcelado, no dividido, y no reduccionista, y el reconocimiento de lo inacabado e incompleto de todo conocimiento aspirando al conocimiento multidimensional.

Experimentar un período traumático impacta en el sujeto más allá del dolor, pues "frena" la construcción de la ética y de la propia estructura neurológica. En pacientes con trastorno de estrés postraumático se han constatado cambios estructurales y/o funcionales en la corteza prefrontal, ACC (cingulado anterior), OFC (orbitofrontal) y amígdala (Francati, Vermetten, \& Bremner, 2007). Áreas comprometidas con el juicio moral. En un estudio reciente con mujeres adultas que experimentaron abusos en la infancia (Nazarov et al., 2016) los investigadores concluyen que la vergüenza, culpa y temor a ser desaprobadas les impedía hacer elecciones utilitaristas. Además, presentaban un déficit de razonamiento altruista asociado a una falta de empatía. Johanna Meehan (1995) expone cómo supervivientes del incesto, principalmente mujeres, sufren de falta de confianza en ellas mismas porque su identidad se ha construido sin respeto a su propio cuerpo. W. Reich (1933) fundamentó en la miseria sexual una de las causas del auge des fascismo.

La traumatización es consecuencia de la violencia que se ejerce contra la víctima por agentes ya aculturados en la violencia. Nuestras sociedades de cultura de "guerra perpetua" y "paz negativa", ejercen una violencia estructural (Galtung, 2003) sobre los sujetos a través de bastantes agentes institucionales. Uno clave es la familia. La traumatización tiene grados de intensidad, casi todos tenemos algo. Una consecuencia es la confusión entre agresividad y violencia, que se convierte en una de las causas profundas de los conflictos, internos y externos. Aristóteles lo tenía claro "...lo violento (bíaios) es contrario a la naturaleza, y posterior a lo que es conforme a ella. De manera que, si en ningún ser corporal hay un movimiento natural, no habrá movimiento de ninguna clase" (Martínez Guzmán, 2001, p. 209). Lo conforme a la naturaleza es la agresividad. El concepto de fuerza de Hannah Arendt (1998, p.147) se refiere a lo mismo. La violencia social es registrada en términos individuales como terror paralizante. Para la psicoanalista Betty. A. Reardon (1996) el origen de la violencia, que justifica el sexismo y la guerra, es el miedo a la diversidad por el temor a perder el poder. Se origina en la infancia en ambos géneros, con matices distintos. El varón se siente vulnerable ante la madre y reacciona con un intento de dominio de lo femenino y cierta fascinación por la guerra. En esta condición se ha perdido el poder comunicativo de actuar concertadamente (Habermas, 1984).

Dos investigadores de la Complutense (Piñuela Sánchez \& Yela García, 2016) han investigado desde la perspectiva (TMT), el efecto de la propia mortalidad (MS) sobre la minimización sentimental (una forma de deshumanización) de los terroristas islamistas, en función de la orientación política de los universitarios estudiados. Concluyen que los resultados apoyan la tesis de que la MS condiciona la actitud más que la posición política.

Hay que precisar que el determinismo no es inexorable, que el conflicto es motor de cambio que aporta la tensión energética necesaria para conciencia y la acción. Esto acerca a la verdad interna y social. "Una de las herramientas más 
utilizadas a nivel internacional, en caso de vulneraciones a los derechos humanos, son las comisiones de la verdad" (Mínguez Alcaide, 2016, p. 33).

Para cambiar la respuesta moral se necesita actuar en la estructura social y en el sujeto. La psicoterapia actúa en el sujeto individualmente o en grupo (Burlingame \& Layne, 2001). Sobre la estructura se necesita tanto deconstruir, para lo que la filosofía, la política, la ciencia, aportan ideas y acciones, como aportar planteamientos que propicien deliberación transformadora. Por citar alguno: las propuestas de reconocimiento elaboradas por Axel Honneth (1997) como alternativas para la transformación pacífica de los conflictos o la de Judith Butler quien con una visión política de deconstrucción antiesencialista, recoge ideas de Austin; de la visión construccionista de la sexualidad de Foucault; de Jacques Derrida; del psicoanálisis lacaniano; de Simone de Beauvoir, para su propuesta performativa de género (Acosta, 2010).

\section{Conclusión y alcance de esta investigación}

Las aportaciones son pocas en relación a la cantidad de preguntas y objeciones suscitadas. Se necesitan más investigaciones de objetivos concretos en una perspectiva consiliente.

Esta la he diseñado desde esa perspectiva, y, por ello, tengo interés en compartirla en la comunidad científica. "A menudo e incluso normalmente, decir algo producirá ciertas consecuencias o efectos sobre los saberes, pensamientos o acciones del auditorio o de quien emite la expresión... Llamamos a la realización de un acto de este tipo un acto performativo (Austin, 1971). Algunos sujetos me comunicaron que el material de la investigación les supuso tomar contacto con actitudes propias desconocidas.

En investigaciones futuras sería conveniente considerar lo siguiente.

Repensar los dilemas como instrumentos válidos. Cabe dudar sobre si el criterio de cantidad es el más adecuado, ¿salvar 6 en vez de uno es más utilitarista? "Un hombre libre en nada piensa menos que en la muerte, y su sabiduría no es una meditación de la muerte, sino de la vida" (Baruch Espinosa en su Ética). Quizás esa sabiduría valorará la utilidad de otra forma por ejemplo asociada a la cualidad. Como mínimo incorporar escenarios más intersubjetivos contemplando el fenómeno de la transferencia.

Incorporar en la investigación sobre el innatismo, aportaciones del psicoanálisis que informan sobre informaciones prepersonales en forma de precogniciones, fantasías inconscientes, y arquetipos colectivos (León-Río, 2009) que prefiguran la capacidad simbólica. De acuerdo con Carl Jung (2002) en la relación con el arquetipo se puede sostener que se necesita la aceptación de acciones "malas", incluso espantosas, que la vida nos invita a cometer en aras de un propósito mayor. Pero solo es válido para la vida si la acción se produce en consonancia con el si-mismo, no con el yo. Esto generará al sujeto un sufrimiento real, al renunciar al ideal de una conciencia pura, en una interacción exigente entre el bien y el mal. El sujeto de la acción sufrirá en su propia alma, sin dejarse vencer por la culpa, pues esta mantendría al sujeto paralizado y en la inercia de la negación de la vida, o podría llevarle a la autocompasión. El sufrimiento es el requisito de veracidad. En términos psicológicos un sujeto debería "sacrificar su yo" para seguir evolucionando. Esto significa matar el vínculo del yo con un narcisismo excluyente del otro, para poder abandonarse al misterio. Ese acto, en soledad radical, es fundante de un funcionamiento complejo, de conciencia sistémico ecológica, de la que el sujeto se siente parte integrante, con humildad y responsabilidad, pues sus actos, siendo autónomos, repercuten en todo el conjunto. Una posición que implica una apertura a lo transpersonal con una responsabilidad moral del sujeto. Una posición muy distinta de la de Abraham. Algunos sujetos de la investigación podrían estar cercanos a ello. Quizás entonces pudiera 
ser real lo que afirma Sartre sobre la libertad "el hombre no tiene naturaleza, no tiene una esencia, por lo que es libre y es lo que él mismo ha decidido ser".

Explorar la "cognición integrada" en funcionamiento de "proceso terciario" (Arieti, Silvano, 1976; Fiorini, Hector J., 1995; Washburn, 1997, 1999). A ese estado, mucho más complejo de funcionamiento, se llega mediante la integración de las diversas facultades y dimensiones humanas, personales y transpersonales. Kohlberg ya había adelantado un estado moral transconvencional con su "etapa cósmica".

Explorar la diversidad antropológica (Garcia, 2017) con una actitud comprometida con los problemas de los seres humanos abandonando el etnocentrismo. Explorar el "relato mítico" que los sujetos se hacen sobre su desarrollo y existencia.

Explorar la relación entre moralidad y capacidad heurística como el arte y la ciencia del descubrimiento y de la invención o de resolver problemas mediante la creatividad y el pensamiento lateral o pensamiento divergente.

Realizar algún ensayo clínico probando alguna intervención que pueda servir para transformar la conciencia moral de los sujetos.

\section{Conflicto de intereses}

El autor declara que no hay conflicto de intereses.

\section{Referencias bibliográficas}

Acosta, C. A. D. (2010). Judith Butler y la teoría de la performatividad de género. Revista de educación y pensamiento, (17), 85-95. Recuperado a partir de https://dialnet.unirioja.es/descarga/articulo/4040396.pdf

Arendt, H. (1998). Sobre la violencia. En Crisis de la república. Madrid: Taurus.

Arieti, Silvano. (1976). La Creatividad: La Síntesis Mágica. Fondo de Cultura Económica.

Austin, J. L. (1971). Palabras y Acciones Como Hacer Cosas Con Palabras. Buenos Aires: Paidós.

Bentham, J. (1983). Deontology; together with a table of the springs of action; and the article on utilitarianism (The Collected Works of Jeremy Bentham). Oxford, England: Oxford University Press. (Original work published 1879).

Blakemore, S.-J., \& Choudhury, S. (2006). Development of the adolescent brain: implications for executive function and social cognition. Journal of Child Psychology and Psychiatry, 47(3-4), 296-312. https://doi.org/10.1111/j.1469-7610.2006.01611.x

Burlingame, G. M., \& Layne, C. M. (2001). Group-based interventions for trauma survivors: Introduction to the special issue. Group Dynamics: Theory, Research, and Practice, 5(4), 243-245. https://doi.org/10.1037/1089-2699.5.4.243

Castano, E., \& Giner-Sorolla, R. (2006). Not Quite Human: Infrahumanization in Response to Collective Responsibility for Intergroup Killing. Journal of Personality and Social Psychology, 90(5), 804-818. https://doi.org/10.1037/0022-3514.90.5.804

Derrida, Jacques. (2000). Dar la muerte. Barcelona: Paidós.

Eliade, M. (1998). El Yoga. Inmortalidad y libertad. México: Fondo de Cultura Económica (1954).

Elster, J., \& Grapes, S. (1982). Utilitarianism and the genesis of wants.

Everett, M. J., Jim A. C. ..Pizarro, David A. ..Crokett. (2016). Inference of Trustworthiness from Intuitive Moral Judgments. Intuitive moral judgments and partner choice. Journal of Experimental Psychology: General, 772-787(145.6), Inglés. Recuperado a partir de http://psycnet.apa.org/journals/xge/145/6/772

Fiorini, Hector J. (1995). Formaciones de procesos terciarios Una tópica del psiquismo creador. Paidós.

Francati, V., Vermetten, E., \& Bremner, J. D. (2007). Functional neuroimaging studies in posttraumatic stress disorder: review of current methods and findings. Depression and Anxiety, 24(3), 202-218. https://doi.org/10.1002/da.20208 
Galtung, J. (2003). Paz por medios pacíficos: paz y conflicto, desarrollo y civilización. Bilbao: Gernika Gogoratuz.

Garcia, M. (2017, enero 24). Amplificación junguiana del mito de surgimiento del yanomami. OSF Open Science Framework. Recuperado a partir de https://osf.io/kxevu

Gray, K. (2014). Harm concerns predict moral judgments of suicide: Comment on Rottman, Kelemen and Young (2014). Cognition, 133(1), 329-331. https://doi.org/10.1016/j.cognition.2014.06.007

Gray, K., Waytz, A., \& Young, L. (2012). The Moral Dyad: A Fundamental Template Unifying Moral Judgment. Psychological Inquiry, 23(2), 206-215. https://doi.org/10.1080/1047840X.2012.686247

Greene, J. D. (2009). The cognitive neuroscience of moral judgment. The Cognitive Neurosciences IV.

Greene, J. D., Sommerville, R., Nystrom, L., Darley, J., \& Cohen, J. (2001). An fMRI investigation of emotional engagement in moral judgment. Science (New York, N.Y.), 293(5537), 2105-2108. https://doi.org/10.1126/science.1062872

Habermas, J. (1984). El concepto de poder en Hannah Arendt. En Perfiles filosófico-políticos. Madrid: Taurus.

Haidt, J., \& Joseph, C. (2007). The moral mind: How 5 sets of innate moral intuitions guide the development of many culture-specific virtues, and perhaps even modules. En P. Carruthers, S. Laurence y S. Stich (Ed.), The Innate Mind (Vol. 3, pp. 367-391). New York: Oxford.

Henwood, K. (2004). Reinventing validity: Reflections on principles and practices from beyond the qualityquantity divide. En Todd, Z., Nerlich, B., Mckeown, S. \& Clarke, D. (Ed.), Mixing methods in psychology (pp. 37-57). Hove: Psychology Press.

Henwood, K. (2008). Qualitative research, reflexivity and living with risk: Valuing and practicing epistemic reflexivity and centering marginality. Qualitative research in psychology, 5(1), 45-55.

Honneth, A. (1997). La lucha por el reconocimiento. Por una gramática moral de los conflictos sociales (Crítica). Barcelona.

Jung, C. G. (2002). Los arquetipos y el inconsciente colectivo (Obra completa, Vol. 9/1). Madrid: Trotta.

Kant, I. (1979). Crítica de la razón pura. México: Porrúa.

Kant, I. (2008). Fundamentación para una Metafísica de las Costumbres. Madrid: Alianza (Trabajo original Publicado en 1785).

Koenigs, M., Kruepke, M., Zeier, J., \& Newman, J. P. (2012). Utilitarian moral judgment in psychopathy. Social Cognitive and Affective Neuroscience, 7(6), 708-714. https://doi.org/10.1093/scan/nsr048

León-Río, B. (2009). Arquetipos e inconsciente colectivo en las artes plásticas a partir de la psicología de C. G. Jung. Arte, individuo y sociedad, (21), 37-50. Recuperado a partir de https://dialnet.unirioja.es/servlet/articulo?codigo=2974800\&orden=211495\&info=link

Lévinas, E. (2000). Ética e infinito (A. Machado Libros, S.A). Madrid.

Maclntyre, A. (1981). After virtue. Londres: A\&C Black Publishers (2013).

Martínez Guzmán, V. (1997). Reconstruir la paz doscientos años después. Una filosofía transkantiana para la paz. En La paz en Kant doscientos años después (pp. 119-134). Valencia: Nau Llibres.

Martínez Guzmán, V. (2001). Filosofía para hacer las paces. Icaria. Recuperado a partir de https://dialnet.unirioja.es/servlet/libro?codigo $=24103$

Mayntz, R., Holm, K., Hübner, P., \& Muñiz, J. N. (1969). Introducción a los métodos de la sociología empírica. Madrid: Alianza Editorial (1993).

Meehan, J. (1995). Feminists read Habermas, Gendering the Subject of Discourse,. New York: Routledge.

Mill, J. S. (1989). El utilitarismo. Madrid: Alianza (Trabajo original en 1863).

Mínguez Alcaide, X. (2016). Apuntes sobre la reparación integral de las vicimas en el contexto vasco. Tolosa: Gotik.

Nazarov, A., Walaszczyk, V., Frewen, P., Oremus, C., Lanius, R., \& McKinnon, M. C. (2016). Moral reasoning in women with posttraumatic stress disorder related to childhood abuse. European Journal of Psychotraumatology, 7. https://doi.org/10.3402/ejpt.v7.31028

Piñuela Sánchez, R., \& Yela García, C. (2016). Mortality salience, political orientation and minimization of terrorists' secondary emotions. Psicothema, 28(1). Recuperado a partir de http://www.redalyc.org/html/727/72743610007/

Reardon, B. (1996). Sexism and the war system. Syracuse University Press. 
Regan, D. (1980). Utilitarianism and Co-operation. Oxford University Press.

Reich, Wilhelm. (1933). Psicología de masas del fascismo (1973). Barcelona: Roca.

Rottman, J., Kelemen, D., \& Young, L. (2014). Purity matters more than harm in moral judgments of suicide: Response to Gray (2014). Cognition, 133(1), 332-334. https://doi.org/10.1016/j.cognition.2014.06.008

Sachdeva, S., Iliev, R., Ekhtiari, H., \& Dehghani, M. (2015). The Role of Self-Sacrifice in Moral Dilemmas. PLOS ONE, 10(6), e0127409. https://doi.org/10.1371/journal.pone.0127409

Scanlon, T. M. (1982). Contractualism and utilitarianism. En A. K. Sen \& B. A. O. Williams (Ed.), Utilitarianism and beyond (pp. 103-110). England: Cambridge University Press. Recuperado a partir de http://www.upscsuccess.com/sites/default/files/documents/Ethical Theory An Anthology @na dal.pdf\#page $=611$

Scanlon, T. M. (1998). What we owe to each other (Vol. 66). Cambridge, MA: Belknap Press of Harvard University Press. Recuperado a partir de https://books.google.es/books?hl=es\&lr=\&id=FwuZcwMdtzwC\&oi=fnd\&pg=PA1\&dq=What+We+ Owe+to+Each+Other\&ots=VVPUpAzDYR\&sig=y 5-OLIhD818Q5MJv xi yXj1N4

Skitka, L. J., \& Sargis, E. G. (2006). The Internet as Psychological Laboratory. Annual Review of Psychology, 57(1), 529-555. https://doi.org/10.1146/annurev.psych.57.102904.190048

Strawson, P. F. (1995). Libertad y resentimiento y otros ensayos (Vol. 37). Grupo Planeta (GBS).

Suchak, M., \& de Waal, F. B. M. (2012). Monkeys benefit from reciprocity without the cognitive burden. Proceedings of the National Academy of Sciences, 109(38), 15191-15196. https://doi.org/10.1073/pnas.1213173109

Washburn, M. (1997). El ego y el fundamento dinámico. Barcelona: Kairós.

Washburn, M. (1999). Psicologia Transpersonal, en una perspectiva psicoanalitica. Barcelona: Los libros de La Liebre de Marzo.

Wellman, H. M., Cross, D., \& Watson, J. (2001). Meta-analysis of theory-of-mind development: the truth about false belief. Child development, 72(3), 655-684. Recuperado a partir de http://onlinelibrary.wiley.com/doi/10.1111/1467-8624.00304/full 
Anexo 1

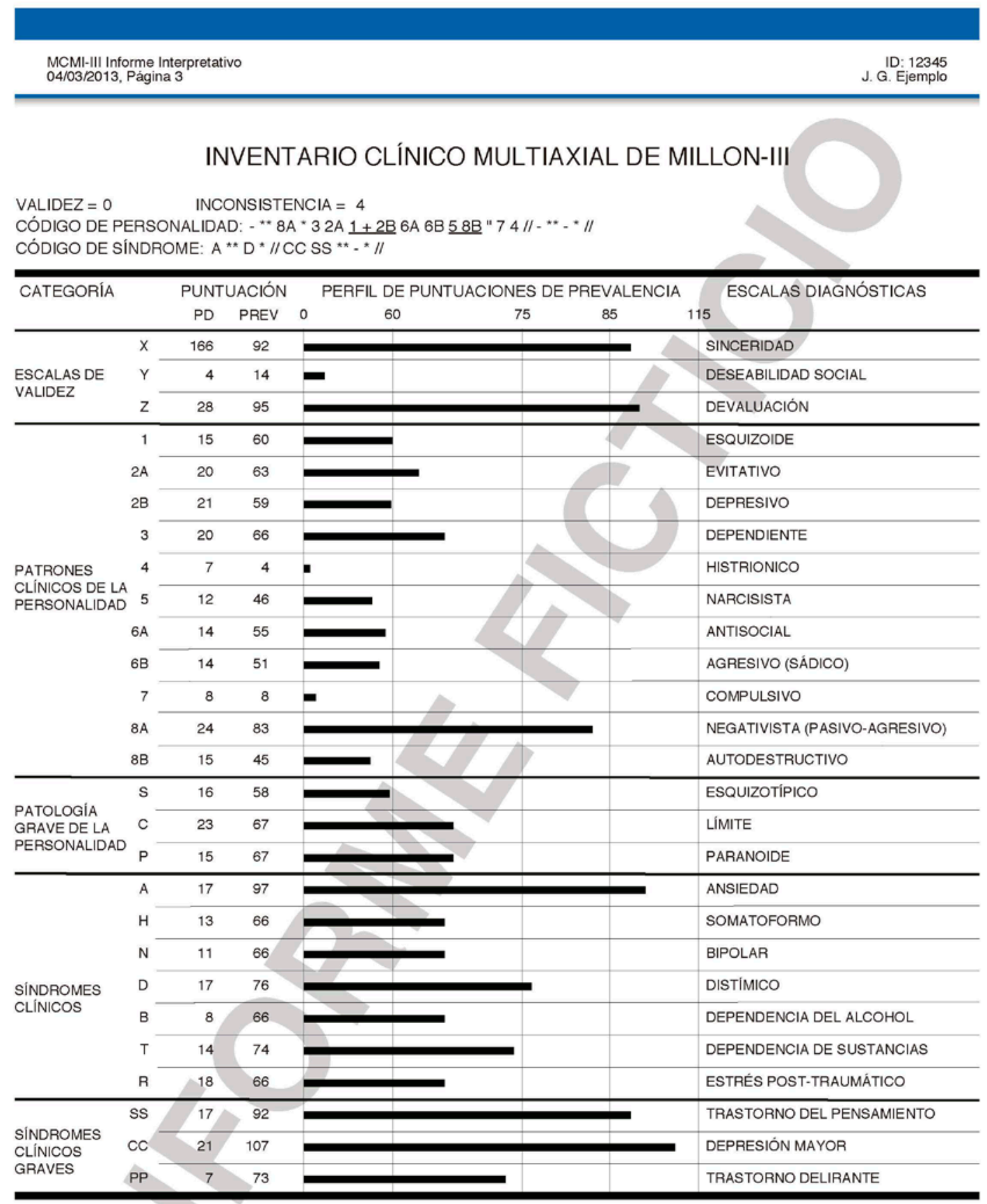

INFORMACIÓN CONFIDENCIAL DE USO EXCLUSIVO DEL PROFESIONAL

Ilustración 5 Perfil gráfico de las escalas de un sujeto ficticio. Extraído de este documento público de $\underline{\text { PEARSON }}$

Sería compatible con un diagnostico DSM-IV: 295.70 Trastorno Esquizoafectivo 300.02 Trastorno de Ansiedad Generalizad 


\section{Anexo 2}

\section{Dilemas morales}

\section{Gratificación}

Un día tienes la oportunidad de elegir entre acostarte una noche con una persona que es famosa y que deseas sexualmente con el compromiso de mantener ese hecho en secreto o pasar con dicha persona un día de modo que además de que seáis vistos en compañía puedas hablar de ello con quien quieras. ¿Qué elegirías?

\section{Demanda causar dolor}

2 Como seguramente sabrás, las personas masoquistas gozan sexualmente sintiendo dolor o humillación. En caso de que convivan con una persona que posea rasgos o tendencias sádicas, sus relaciones son satisfactorias para los dos miembros de la pareja, y las repercusiones de ese dolor placentero son asumibles, en términos generales, para ambos.

El problema sexual aparece cuando uno de los dos miembros de la pareja no goza con tales actos. Por ejemplo, un individuo que conviva con una persona masoquista se ve en el dilema de causar dolor (algo que en sí mismo le repugna moralmente) para que su pareja goce.

Si tú te encontraras en una situación de ese tipo, ¿accederías a causar dolor a la persona a la que quieres o decidirías romper con ella a pesar de tu amor por creer que estás cometiendo acciones inmorales?

\section{Tirar al moribundo}

\section{El hombre de pie sostiene a un moribundo. Si tu estuvieras en esa situación clo tirarías al agua?}

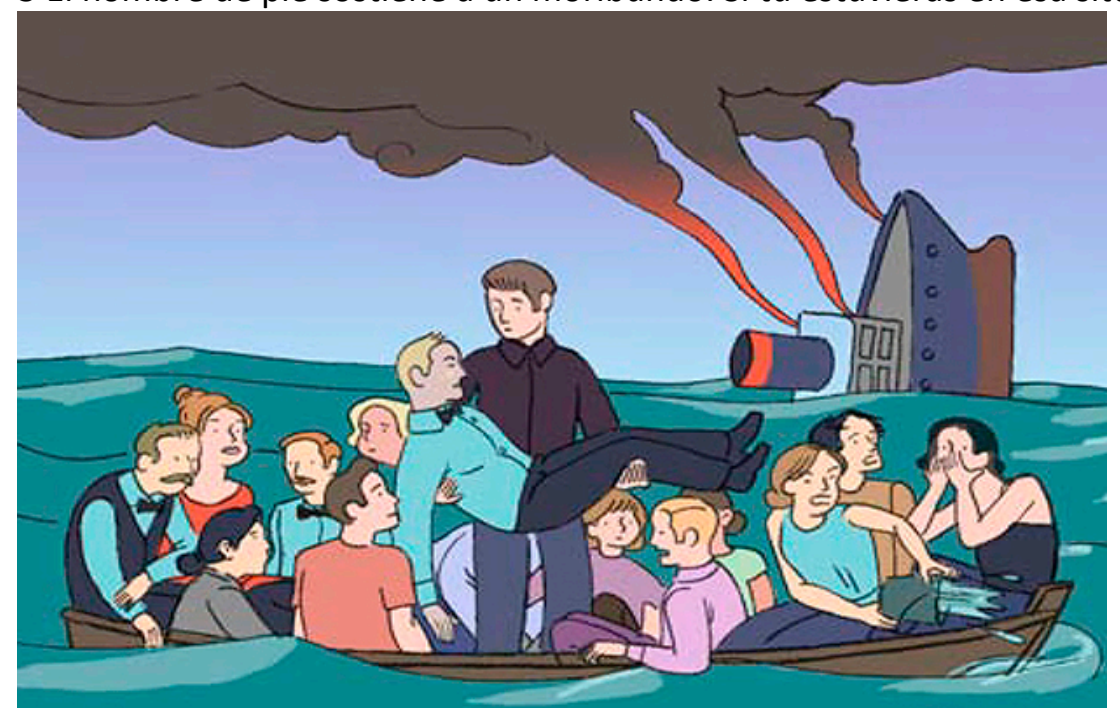

\section{Perdón}

4 La emoción es una reacción psíquica que altera el equilibrio y el comportamiento racional de cualquier persona. Sin embargo, existen individuos capaces de dominarla, y nunca (o casi nunca) pierden el control de sus actos, mientras otros, en cambio, se dejan llevar por ella y realizan, bajo su efecto, acciones de las que posteriormente se arrepienten.

Imagínate que tu pareja, arrastrada por la emoción y el cariño que profesa a un antiguo/a amigo/a, y ante la insistencia de éste/a, apelando al afecto que aún existe entre los dos, comete un acto de infidelidad para contigo.

¿Lo/a perdonarías por el hecho de haber actuado con una gran carga emotiva? ¿O no lo harías por creer que podía haber controlado su emoción antes de realizar un acto que traicionaba vuestra mutua confianza?

\section{5 y 6 . Excluidas de esta investigación.}




\section{Autorizar inseminación}

7 Suponte que eres juez y en tu juzgado entra una demanda de una mujer que solicita utilizar el semen de su marido muerto para tener un hijo mediante inseminación artificial. Tú ignoras cuál era la voluntad del muerto al congelar su semen (podría ser, por ejemplo, para tener descendencia con otra mujer diferente a la suya), ya que sólo tienes como referencia la palabra de su esposa, sin que puedas contrastarla con ninguna otra.

Se te plantea el dilema de autorizar o denegar la inseminación artificial, basándote únicamente en razones éticas, ya que la ley no contempla específicamente un caso como éste. ¿Qué harías y cuál sería tu justificación?

\section{Altruismo/propio interés.}

8 Imagínate que buscas trabajo y te contratan en una industria que se dedica a la elaboración de productos químicos. Al cabo de los meses compruebas, por tu propia experiencia, que la empresa no respeta la legislación medioambiental vigente y está contaminando las aguas de un río con los residuos de una sustancia química que pasa desapercibida a simple vista y cuyos efectos no pueden ser comprobados a corto plazo, aunque sí a largo.

Esto te plantea un dilema de difícil resolución: ¿debes denunciar a la empresa por causar contaminación, o debes guardar silencio para garantizar tú puesto de trabajo e incluso el de tus compañeros (te podrían despedir, o si la multa y los costes para adaptarse a la legislación ecologista fuesen muy altos la empresa podría cerrar)? ¿Qué podría más en este caso concreto: tú interés ecológico o tu interés personal por el trabajo? 